\title{
Title
}

\section{Kinetochore-localized PP1/Sds22 couples chromosome segregation to polar relaxation}

\section{Authors}

Nelio T.L. Rodrigues ${ }^{1}$, Sergey Lekomtsev ${ }^{1}$, Silvana Jananji ${ }^{2}$, Janos Kriston-Vizi ${ }^{1}$, Gilles R.X. Hickson ${ }^{2,3}$ and Buzz Baum ${ }^{1,4,5^{*}}$

\section{Affiliations}

1 - MRC Laboratory for Molecular Cell Biology,

University College London, Gower St, London, WC1E 6BT, UK.

2 - Sainte-Justine Hospital Research Center, Montréal, Québec, Canada.

3 - Dept. Pathology \& Cell Biology, Université de Montréal, Montréal, Québec, Canada.

4 - Institute for the Physics of Living Systems, University College London, Gower St, London, WC1E 6BT, UK.

5 - CelTisPhyBio Labex, Institut Curie, 26 rue d'Ulm 75248 Paris cedex 05.

* Corresponding author 


\section{Summary}

Cell division requires the precise coordination of chromosome segregation and cytokinesis. This coordination is achieved by the recruitment of an actomyosin regulator, Ect2, to overlapping microtubules at the center of the elongating anaphase spindle $^{1}$. Ect 2 then signals to the overlying cortex to promote the assembly and constriction of an actomyosin ring between the segregating chromosomes ${ }^{1}$. Here, by studying division in proliferating animal cells we demonstrate the existence of a second, parallel signaling pathway that triggers the relaxation of the polar cell cortex at mid-anaphase. This is independent of furrow formation, centrosomes and microtubules and, instead, depends on PP1 phosphatase and its regulatory subunit $\operatorname{Sds} 22^{2,3}$. As separating chromosomes move towards the polar cortex at mid-anaphase, kinetochore-localized PP1/Sds22 helps to break cortical symmetry by inducing the dephosphorylation and inactivation of ERM proteins (Ezrin/Radixin/Moesin) at cell poles. This promotes local softening of the $\operatorname{cortex}^{2,3}$, facilitating anaphase elongation and orderly cell division. In sum, this identifies a conserved kinetochore-based phosphatase signal and substrate, which function together to link anaphase chromosome movements to cortical polarization - coupling chromosome segregation to cell division. 


\section{Main text}

As animal cells exit mitosis they assemble a cleavage furrow at their equator, whose position is determined by the overlapping microtubules of the central spindle ${ }^{1}$. This ensures the precise coordination of cell division and chromosome segregation. Recently, however, it has become clear that proliferating animal cells assemble a rigid and isotropic actomyosin cortex as they enter mitosis ${ }^{4}$ which, if not disassembled, might interfere with furrow formation. To test this idea, and to determine whether relaxation of the cortex at opposing cell poles contributes to cell division ${ }^{5}$, we analyzed sensory organ precursor (SOP) cells in the Drosophila pupal notum, where live-cell imaging can be used to study the changes in cell shape that accompany mitotic progression in the context of a developing epithelium ${ }^{6,7}$. In these cells, actin was cleared from cell poles before any overt signs of equatorial constriction were visible (Fig.1a-b); as previously proposed ${ }^{8}$. Similarly, polar relaxation was found to precede furrow formation in human cells (Fig.1c). Moreover, in both systems, polar relaxation, as evidenced by actin clearance, cell elongation and/or blebbing, was not affected by silencing of the centralspindlin component RacGAP1 (MgcRacGAP/Tumbleweed) (Fig.1d, Extended Data Fig.1a, d and Extended Data Fig.2a-b), even though this led to profound defects in the equatorial recruitment of actin and myosin-II, furrow formation and cytokinesis (Extended Data Fig.1a-c, e, Extended Data Fig.2c-d) (100\% of human RacGAP1-depleted cells failed to divide $^{9,10}$, as did $30 \%$ of Tumbleweed RNAi cells in the fly). In addition, actin (Extended Data Fig.2e-g) and phosphoT559-Moesin (p-Moesin) (Extended Data Fig.2h) disappeared from the polar cortex prior to their accumulation at the furrow. Together, these data imply the existence of a conserved second signal that functions independently of the well-studied spindle midzone to polarize the anaphase cortex.

Having shown that anaphase polar relaxation is independent of furrow formation it was important to identify the source of the signal. We began by considering the possibility that the signal is mediated by centrosomes and associated astral microtubules, since they have previously been proposed to regulate cortical

contractility at the cell equator ${ }^{11,12}$ and at cell poles ${ }^{13,14}$. We eliminated both through mutation of the gene asterless ${ }^{15}$ (Extended Data Fig.3a-c). This led to randomization of the orientation of the spindle (Extended Data Fig.3d) without compromising 
mitotic progression, as expected following a loss of crosstalk between the metaphase spindle and cortex. Nevertheless, in anaphase, both F-actin (Fig.1e-f and Extended Data Fig.3e) and p-Moesin (Fig.1g-h) were cleared from opposing cell poles as efficiently as they were in control cells.

As an alternative approach to address the same question, we used a CDK inhibitor (RO3306) to force cultured cells to undergo a monopolar cytokinesis ${ }^{16}$. As cortical symmetry was broken under these conditions, p-ERM was lost from the cortex on the side of the cell distal from centrosomes and most microtubules (Extended Data Fig.3f). Similar results were observed in cells forced to exit mitosis in the presence of high doses of microtubule inhibitors following either CDK inhibition (Extended Data Fig.3g-i, k) or Mad2 depletion (Extended Data Fig.3g-h, j-k). These data show that the cortical polarization induced by mitotic exit is independent of both centrosomes and/or astral microtubules. Strikingly, however, actin filaments and p-ERM proteins were always lost from portions of the cortex closest to anaphase chromatin (Extended Data Fig.3f-k). To assess the timing of this chromosomally-associated signal, we repeated the analysis in cells forced to flatten through the expression of an activated version of the Rap1 GTPase $(\operatorname{Rap} 1 *)^{17}$. Although this pushed metaphase chromosomes close to the cell cortex, CDK inhibition again induced a rapid loss of local F-actin and cortical relaxation (Extended Data Fig.4a-b), implying an enhancement of the signal at the onset of anaphase.

To see whether segregating chromosomes perform a similar function during a normal mitotic exit (Fig2.a and Supplementary Video 1), we generated kymographs of dividing SOP cells (Fig.2b) to monitor the relative timing of anaphase cell elongation (D1), the distance of the chromatin (kinetochore interface) to the cell center (D2), and the approach of chromosomes to the poles (D3) (Fig.2c and Extended Data Fig.4c-d). Under these physiological conditions the approach of the chromatin mass to cell poles was associated with loss of cortical actin (Fig.2d-e), which translated into a simple, near-linear relationship between the distance of kinetochores from the cell pole and cortical actin clearance (Fig.2f-h). Further, in SOP cells, cortical actin was lost from the anterior pole first, in line with this being the first to come into close proximity with chromatin (Extended Data Fig.4f-i). Taken together, these data support the 
existence of a chromosomally-derived signal that induces polar relaxation following mitotic exit.

PP1-87B phosphatase and its regulatory subunit, Sds22 seemed good candidates for molecular regulators of this process since previous work showed that they negatively regulate $\mathrm{p}$-Moesin during mitotic exit ${ }^{2,3}$. As previously reported ${ }^{2}$, RNAi mediated silencing of either PP1-87B or Sds22 led to a failure in clearance of the polar cortex at anaphase (Fig.3a-b and Supplementary Videos 2-3). This was accompanied by a defect in cell elongation (Extended Data Fig.5c-h), enabling anaphase chromosomes to come much closer to the cortex of PP1-87B or Sds22 RNAi cells than they did in the control (Fig.3a, Extended Data Fig.5a-b; a similar phenotype was observed in cultured cells depleted for Sds22 (Fig.3c-d and Extended Data Fig.6a-d)). Importantly, these defects were recapitulated by the expression of a constitutively active, phospho-mimetic form of Moesin (Extended Data Fig.7a-f), suggesting that pMoesin is a key target of the phosphatase holo-enzyme. Moreover, PP1-87B complexes immunoprecipitated from fly cells efficiently dephosphorylated activated Moesin in a manner that depended on Sds22 (Extended Data Fig.7g-h). The block in polar relaxation induced by $\mathrm{PP} 1 / \mathrm{Sds} 22$ silencing or the expression of constitutively active Moesin led to a near-identical series of extreme contortions as cells entered telophase (Extended Data Fig.8a-d and Supplementary Videos 4-7). Although these appeared to compromise division, cells nearly always completed a successful cytokinesis. This we attribute to the parallel recruitment of myosin-II to the midzone, which is robust and functionally independent of PP1/Sds22 ${ }^{18}$ (Extended Data Fig.9ac).

To determine the likely localization of the relevant PP1/Sds22 signal, we used an Sds22-GFP transgene to study the dynamic localization of PP1/Sds22 during mitotic progression. Several PP1 complexes are found at kinetochores, where they counteract Aurora B activity and silence the mitotic checkpoint ${ }^{19-21}$. Some, like Repo-Man/PP1, have been reported to move from the centromeres to chromosome arms at the metaphase-anaphase transition ${ }^{22}$. However, Sds22-GFP was found concentrated at kinetochores (Extended Data Fig.10a) during both metaphase and anaphase (Fig.4a and Extended Data Fig.10a-c). Moreover, the approach of kinetochore-bound Sds22GFP to the cortex in mid-anaphase was associated with the induction of local 
blebbing (Fig.4a-b and Extended Data Fig.10b-c). To determine whether this midanaphase polar relaxation depends on the observed accumulation of PP1/Sds22 at kinetochores, we followed the process in KNL1 RNAi cells, where the kinetochore recruitment of PP1/Sds22 is compromised ${ }^{23}$. Strikingly, the resulting drop in kinetochore-localisation of Sds22 (as measured by Sds22-GFP, Fig.4a and Extended Data Fig.10d-e) was associated with the loss of polar blebbing and with reduced anaphase cell elongation (Fig.4b-c).

Finally, to test whether a spatially-defined PP1 phosphatase signal is sufficient to trigger local relaxation of the cortex, as predicted by this model, we used optogenetics $^{24}$ to artificially target the subunit Sds22 to the plasma membrane in cells arrested in mitosis. By fusing cryptochrome 2 (CRY2) to Sds22 (CRY2-mCherrySds22), we were able to use light to drive the phosphatase to the membrane in cells co-expressing membrane-tethered CIBN $^{24}$ (see Methods section) (Extended Data Fig.10f). In more than half of the cells analyzed, the local recruitment of Sds22mCherry was associated with bleb formation (Fig.4d). Thus, PP1/Sds22 is both required for anaphase cortical relaxation and is sufficient to drive this process when mislocalised to the cortex during metaphase.

In summary, while work over many decades has focused on crosstalk between the central spindle and the overlying actomyosin cortex in positioning the cleavage furrow $^{1,9,18}$, this study reveals an additional, conserved kinetochore-based PP1/Sds22 signal that helps to couple chromosome segregation to anaphase cell elongation. Both signals likely act in parallel to generate the gradient in cortical actomyosin contractility required for cytokinesis $^{25}$ (Fig.4e). The localization of PP1/Sds22 to kinetochores is striking, since related PP1 phosphatase complexes act at the same site to help control the decision to exit mitosis and to ensure the stable attachment of chromosomes to kinetochore microtubules ${ }^{19-21}$. As is the case for the PP1-mediated stabilization of MT-kinetochore attachments ${ }^{20}$, the ability of kinetochore-localized PP1/Sds22 to act on the cortex likely depends on the phosphatase complex counteracting the activity of Aurora kinases, since this would explain the ability of Sds22 to induce cortical relaxation when forcibly mislocalised to the plasma membrane in metaphase. Unlike other phosphatase subunits ${ }^{22}$, however, Sds22-GFP remains on kinetochores throughout anaphase. Importantly, this places the PP1/Sds22 
phosphatase complex in a perfect position to mediate communication between segregating chromosomes and the anaphase cortex, at least in part, through the local dephosphorylation of ERM proteins at cell poles. While recent studies have suggested a similar role for chromatin-bound Ran-GTP in cortical polarisation during both meiotic and mitotic divisions ${ }^{26,27}$ (Fig.4e), the mechanistic link between Ran and the actomyosin cortex is not clear. Moreover, a function for Ran in this process is hard to reconcile with the enhancement of the polar relaxation signal seen following mitotic exit (Extended Data Fig.4a-b). Therefore more work is required to determine how Ran, PP1/Sds22 and other signals emanating from the anaphase spindle function together to break cortical symmetry at mitotic exit, and how this initial difference between cortical contractility at cell poles and the midzone is amplified to drive furrow formation and cytokinesis, during both symmetric and asymmetric animal cell divisions. 


\section{Acknowledgements}

NR, SL and BB thank Cancer Research UK, and JKV the Medical Research Council for funding. SJ and GH were funded by the Canadian Institutes of Health Research, the Canada Foundation for Innovation and a salary award from the Fonds de Recherche du Québec-Santé, and GH thanks the Cole Foundation for a Transition award. This study benefited from support from INCa and the BBSRC (BB/K009001/1). We thank M. Lam, E. Paluch, M. Petronczki, G. Salbreux and members of the Baum lab for input and critical reading of the manuscript.

\section{Author Contributions}

NR designed and conducted all experiments using Drosophila flies and helped analyse human cell data with the aid of JKV. SL designed and conducted all experiments using human cells. SJ and GH conducted all experiments in fly cell culture. BB oversaw the project, which was conceived by NR and BB. NR, SL and $\mathrm{BB}$ wrote the manuscript.

\section{Competing Financial Interests}

The authors declare no competing financial interests. 


\section{Main References}

1 Green, R. A., Paluch, E. \& Oegema, K. Cytokinesis in animal cells. Annu Rev Cell Dev Biol 28, 29-58, doi:10.1146/annurev-cellbio-101011-155718 (2012).

2 Kunda, P. et al. PP1-mediated moesin dephosphorylation couples polar relaxation to mitotic exit. Curr Biol 22, 231-236, doi:S0960-9822(11)01391-1 [pii]

10.1016/j.cub.2011.12.016 (2012).

3 Roubinet, C. et al. Molecular networks linked by Moesin drive remodeling of the cell cortex during mitosis. J Cell Biol 195, 99-112, doi:jcb.201106048 [pii] 10.1083/jcb.201106048 (2011).

4 Matthews, H. K. et al. Changes in Ect2 localization couple actomyosindependent cell shape changes to mitotic progression. Dev Cell 23, 371-383, doi:S1534-5807(12)00276-6 [pii]

10.1016/j.devcel.2012.06.003 (2012).

5 Sedzinski, J. et al. Polar actomyosin contractility destabilizes the position of the cytokinetic furrow. Nature 476, 462-466, doi:nature10286 [pii]

10.1038/nature10286 (2011).

6 Roegiers, F., Younger-Shepherd, S., Jan, L. Y. \& Jan, Y. N. Two types of asymmetric divisions in the Drosophila sensory organ precursor cell lineage. Nat Cell Biol 3, 58-67, doi:10.1038/35050568 (2001).

7 Georgiou, M. \& Baum, B. Polarity proteins and Rho GTPases cooperate to spatially organise epithelial actin-based protrusions. J Cell Sci 123, 10891098, doi:jcs.060772 [pii]

10.1242/jcs.060772 (2010).

8 Hickson, G. R., Echard, A. \& O'Farrell, P. H. Rho-kinase controls cell shape changes during cytokinesis. Curr Biol 16, 359-370, doi:S09609822(06)01026-8 [pii]

10.1016/j.cub.2005.12.043 (2006).

9 Mishima, M., Kaitna, S. \& Glotzer, M. Central spindle assembly and cytokinesis require a kinesin-like protein/RhoGAP complex with microtubule bundling activity. Dev Cell 2, 41-54, doi:S1534580701001101 [pii] (2002).

10 Lekomtsev, S. et al. Centralspindlin links the mitotic spindle to the plasma membrane during cytokinesis. Nature 492, 276-279, doi:nature11773 [pii]

10.1038/nature11773 (2012). 
11 Tse, Y. C., Piekny, A. \& Glotzer, M. Anillin promotes astral microtubuledirected cortical myosin polarization. Mol Biol Cell 22, 3165-3175, doi:mbc.E11-05-0399 [pii]

10.1091/mbc.E11-05-0399 (2011).

12 Fededa, J. P. \& Gerlich, D. W. Molecular control of animal cell cytokinesis. Nat Cell Biol 14, 440-447, doi:ncb2482 [pii]

$10.1038 /$ ncb2482 (2012).

13 Murthy, K. \& Wadsworth, P. Dual role for microtubules in regulating cortical contractility during cytokinesis. J Cell Sci 121, 2350-2359, doi:jcs.027052 [pii]

10.1242/jcs.027052 (2008).

14 Werner, M., Munro, E. \& Glotzer, M. Astral signals spatially bias cortical myosin recruitment to break symmetry and promote cytokinesis. Curr Biol 17, 1286-1297, doi:S0960-9822(07)01644-2 [pii]

10.1016/j.cub.2007.06.070 (2007).

15 Blachon, S. et al. Drosophila asterless and vertebrate Cep152 Are orthologs essential for centriole duplication. Genetics 180, 2081-2094, doi:genetics.108.095141 [pii]

10.1534/genetics.108.095141 (2008).

16 Hu, C. K., Coughlin, M., Field, C. M. \& Mitchison, T. J. Cell polarization during monopolar cytokinesis. J Cell Biol 181, 195-202, doi:jcb.200711105 [pii]

10.1083/jcb.200711105 (2008).

17 Lancaster, O. M. et al. Mitotic rounding alters cell geometry to ensure efficient bipolar spindle formation. Dev Cell 25, 270-283, doi:S15345807(13)00185-8 [pii]

10.1016/j.devcel.2013.03.014 (2013).

18 Zhang, D. \& Nicklas, R. B. 'Anaphase' and cytokinesis in the absence of chromosomes. Nature 382, 466-468, doi:10.1038/382466a0 (1996).

19 Lesage, B., Qian, J. \& Bollen, M. Spindle checkpoint silencing: PP1 tips the balance. Curr Biol 21, R898-903, doi:S0960-9822(11)00969-9 [pii]

10.1016/j.cub.2011.08.063 (2011). 
20 Posch, M. et al. Sds22 regulates aurora B activity and microtubulekinetochore interactions at mitosis. J Cell Biol 191, 61-74, doi:jcb.200912046 [pii]

10.1083/jcb.200912046 (2010).

21 Wurzenberger, C. et al. Sds22 and Repo-Man stabilize chromosome segregation by counteracting Aurora B on anaphase kinetochores. J Cell Biol 198, 173-183, doi:jcb.201112112 [pii]

10.1083/jcb.201112112 (2012).

22 Qian, J., Lesage, B., Beullens, M., Van Eynde, A. \& Bollen, M. PP1/Repoman dephosphorylates mitotic histone $\mathrm{H} 3$ at $\mathrm{T} 3$ and regulates chromosomal aurora B targeting. Curr Biol 21, 766-773, doi:S0960-9822(11)00351-4 [pii]

10.1016/j.cub.2011.03.047 (2011).

23 Liu, D. et al. Regulated targeting of protein phosphatase 1 to the outer kinetochore by KNL1 opposes Aurora B kinase. J Cell Biol 188, 809-820, doi:jcb.201001006 [pii]

10.1083/jcb.201001006 (2010).

24 Kennedy, M. J. et al. Rapid blue-light-mediated induction of protein interactions in living cells. Nature methods 7, 973-975, doi:10.1038/nmeth.1524 (2010).

25 Turlier, H., Audoly, B., Prost, J. \& Joanny, J. F. Furrow constriction in animal cell cytokinesis. Biophys J 106, 114-123, doi:S0006-3495(13)01244-7 [pii]

10.1016/j.bpj.2013.11.014 (2014).

26 Kiyomitsu, T. \& Cheeseman, I. M. Cortical dynein and asymmetric membrane elongation coordinately position the spindle in anaphase. Cell 154, 391-402, doi:S0092-8674(13)00712-5 [pii]

10.1016/j.cell.2013.06.010 (2013).

27 Dehapiot, B. \& Halet, G. Ran GTPase promotes oocyte polarization by regulating ERM (Ezrin/Radixin/Moesin) inactivation. Cell cycle 12, 1672-1678, doi:10.4161/cc.24901 (2013). 


\section{Figure Legends}

Figure 1 - Polar relaxation and anaphase cell elongation are independent of furrow ingression and centrosomes/astral microtubules.

a-b, Representative stills and graph to show SOP cell elongation during mitotic exit, before and after furrowing (18 cells). A/P denotes anterior-posterior axis. c, Equivalent HeLa cell imaged at mitotic exit (representative of 10 biological replicates). d (left and right), Representative DIC stills for 26 control and 22 RacGAP1-depleted anaphase cells, together with quantification of cell elongation in each case relative to metaphase. e-f, Levels of polar actin in early and mid-anaphase in 12 control and $16 \mathrm{Asl}^{\mathrm{mecD}}$ SOP cells (e shows representative images of cells quantified in f). g-h, Ratio poles/equator of cortical p-Moesin levels in immunostained fly cells ( $\mathrm{g}$ shows representative image of cells quantified in $\mathrm{h}$ ); White arrowheads in a and c indicate polar relaxation; F-actin is labelled with Lifeact-GFP and by GMA-GFP in e. Asterisks mark chromosomes. Anaphase onset $=0 \mathrm{sec}$. Scale bars $=5 \mu \mathrm{m}$. Bar graphs show mean and standard deviation; box-and-whisker plots median and 10-90 percentiles. Significance in each case was assessed using a twotailed unpaired t-test.

Figure 2 - Polar relaxation is triggered by chromatin proximity in midanaphase.

a-b, Images and the corresponding kymograph for SOP cell undergoing anaphase (representative of 12 biological replicates). Arrowheads indicate polar relaxation. Anaphase onset $=0$ sec. c, Scheme depicting distances D1 and D3. d-e, Show distances D1, D3 (d) and relative actin levels (e) before and after the onset of elongation. f, Scheme of cortical regions c1-9 and DNA-to-cortex distances d1-9. g14, Kymographs of cortical actin and DNA-to-cortex distance over time for a representative SOP cell (g1-g2), and when averaged over 10 cells (g3-g4). h, Actin levels (seen in $\mathrm{g} 3$ ) were strongly correlated $\left(\mathrm{r}^{2}=0.83\right)$ with DNA-to-cortex distance (seen in g4); F-actin was labeled with Lifeact-GFP. Scale bars $=5 \mu \mathrm{m}$. Graphs show mean with standard deviation. Significance was assessed using a two-tailed unpaired t-test. 
Figure 3 - PP1-87B and Sds22 phosphatase subunits are required for actin clearance and polar relaxation at mid-anaphase.

a-b, Polar actin clearance was analysed in control, PP1-87B RNAi and Sds22 RNAi SOP cells undergoing anaphase (images are representative of 15/15/14 cells respectively). Arrowheads in a, indicate polar relaxation. Anaphase onset $=0 \mathrm{sec}$. Scale bar $=5 \mu \mathrm{m}$. b, Kymographs for anterior pole of cells shown in a. Arrowhead indicates elongation onset. Gray box highlights polar relaxation (or impairment thereof). No significant clearance of polar actin was observed in PP1-87B-depleted cells ( $p=0.099,16$ cells) or Sds22-depleted cells $(p=0.141,10$ cells) (as measured in Fig.2e). c-d, XZ cross-sections of STLC-treated control (representative of 26) or Sds22 RNAi (representative of 23) HeLa cells expressing Rap1* before and after RO3306 addition, together with quantification of actin clearance from the cortex above the DNA. Green arrowhead points to actin-enriched cortex. White arrowhead points to actin-devoid cortex. Scale bars $=10 \mu \mathrm{m}$. Lifeact-GFP labels F-actin in a-c. Median is shown in scatter plot. Significance was assessed using a two-tailed unpaired t-test.

Figure 4 - Cortical relaxation is induced by the local accumulation of Sds22.

a-c, Cortical relaxation in Sds22-GFP-expressing fly cells in control and KNL1depleted backgrounds. a, representative images, together with $\mathbf{b}$, their outlines over time, and c, a graph of cell elongation showing mean and standard deviation. Inset regions denoted by green arrowheads. Black arrowhead points to polar blebbing. Asterisks mark DNA. Anaphase onset $=0 \mathrm{sec}$. d, Representative images of HEK293 T cells expressing CRY2-mCherry (31 cells), CRY-mCherry-Sdss22 (39 cells) or CRY2-mCherry/Sds22-GFP (25 cells) following localised illumination with blue light (blue box). e, Model showing three anaphase signals that induce polarization of the actomyosin cortex: 1 - Kinetochore-localised PP1/Sds22 inactivates Moesin and triggers polar relaxation. 2 - Spindle midzone promotes equatorial accumulation of myosin-II and furrowing. 3 - Chromatin-derived RanGTP is proposed to induce cortical repolarization and polar expansion. Scale bars $=5 \mu \mathrm{m}$. Graphs show mean with standard deviation. Significance was assessed using a two-tailed unpaired t-test. 


\section{Methods}

\section{Drosophila strains and husbandry.}

The following fly transgenes/alleles were used: neur-RFP (from Y. Bellaiche), UASLifeAct::GFP (from F. Schnorrer), pnr-GAL4 (BL3039), EM462-GAL4 (from G. Morata), tub-GAL80 ${ }^{\text {ts }}$ (BL7018 and BL7108), neur-GMA (GFP fused to the F-actinbinding domain of the fly Moesin ${ }^{28}$, expressed under the neuralized promoter), Sqh::mCherry (from E. Wieschaus), ubi-RFP::Centrosomin (from J. Raff), Asl ${ }^{\text {mecD }}$ (from J. Raff), gSpc25::mRFP (from C. Lehner), UAS-Sds22::GFP (from B. Thompson) and UAS-Moesin::GFP and UAS-MoesinT559D::GFP (from F. Payre). RNAi lines were used to silence the expression of the following genes: RacGAP1/tumbleweed (DRSC, HMS01417), Sds22 (VDRC, TID42051), PP1-87B (VDRC, TID35024) and KNL1/Spc105R (DRSC, HMS01752). To drive RNAi expression, we first generated a viable fly strain encoding neur-RFP (which labels the chromatin of sensory organ cells at all stages of the cell cycle), pnr-GAL4 (pannier is expressed in the central region of the notum ${ }^{29}$ ) and UAS-LifeAct::GFP (which enables visualization of actin filaments). Female $\mathrm{s}$ of this line were then crossed to UAS-hairpin RNAi males. Expression of the inducible hairpin targeting either PP1-87B or RacGAP1 resulted in high lethality during early development. To overcome that, a GAL80 temperature sensitive transgene was used ${ }^{30}$. All flies were grown at $18^{\circ} \mathrm{C}$ and shifted to $29^{\circ} \mathrm{C}$ (at which temperature GAL80 is inactive, allowing GAL4-dependent gene transcription) at the beginning of pupariation. Expression of the inducible hairpin targeting KNL1/Spc105R was accomplished using the EM462-GAL4 driver.

\section{Cell lines.}

Well-established cell lines were used, all of which have been used in previously published studies from Baum and Hickson labs and their collaborators. Human cell lines were subjected to frequent mycoplasma tests and tested negative relative to a positive control.

\section{Plasmids for expression in human cells.}

To create Cry2-mCherry-Sds22, the Sds22 coding sequence (NM_002712) was inserted into Cry2-mCherry by using sequence- and ligation-independent cloning 
(SLIC) as described ${ }^{31}$. Briefly, Cry2-mCherry vector was linearized by inverse PCR reaction. Sds 22 was amplified by PCR with primers containing $20 \mathrm{bp}$ extension homologous to each end of linearized vector. Vector and insert were mixed and incubated with T4 polymerase followed by transformation in TOP10 competent cells (Invitrogen).

\section{siRNA transfection.}

The following siRNA duplexes were used at a final concentration of $50 \mathrm{nM}$ : AllStars control (Qiagen, 1027280), RacGAP1 (Invitrogen Stealth HSS120934) (GCCAAGAACUGAGACAGACAGUGUG) and Sds22 (Qiagen Hs_PPP1R7_5) (CCAGATCAAGAAGATTGAGAA). Lipofectamine RNAiMAX (Invitrogen) was used for siRNA transfection. Cells were analysed $31 \mathrm{~h}$ after transfection with siRNA. For the experiments of cell flattening (in Fig.3c-d, Extended Data fig.4a-b and Extended Data fig.6d), cells were transfected with Rap1* prior to siRNA treatment, as previously described ${ }^{17}$.

\section{Live imaging of Drosophila SOP cells.}

For time-lapse imaging, fly pupae were first attached to a slide with double-sided tape on their ventral side, as described before ${ }^{7}$. A small window was then cut in the pupal case on the dorsal side, thereby exposing the notum. A coverslip with a drop of halocarbon oil was then placed over the whole fly, supported by coverslips glued onto either end of the slide to allow imaging on confocal microscopes. Time-lapse movies were acquired using a Leica SPE confocal microscope with a 40X lens (NA 1.3). Nota were imaged at 14-16 h after puparium formation (APF). In all cases, male and female flies were treated equivalently without randomization; animal numbers used were determined by experimental constraints.

\section{Drosophila S2 cell experiments.}

Schneider's S2 cells (from O'Farrell lab, UCSF) grown in Schneider's medium supplemented with $10 \%$ fetal calf serum (Life Technologies) were seeded in 8-well Labtek chambered coverglass dishes (Thermo Fisher Scientific) and treated with 25 $\mu \mathrm{M}$ colchicine (Sigma) for $30 \mathrm{~min}$ prior to the start of imaging or fixation. Mitotic exit was induced by prior incubation for $48 \mathrm{~h}$ with Mad2 dsRNAs (as previously described $^{32}$ ) or by $10 \mu \mathrm{M}$ RO3306 for 5-10 min. Cells were fixed using $4 \%$ 
formaldehyde in PBS for 10 min prior to permeabilisation and blocking with PBS containing $0.1 \%$ triton X-100 and 5\% normal goat serum (Jackson ImmunoResearch). Cells were immunostained using antibodies against p-ERM (Cell Signaling, 3141S, 1/200) and Anillin (1/1000, a gift from C. Field). DNA was stained using Hoechst 33258 (1/1000) and F-actin was stained with Alexa ${ }^{546}$-conjugated phalloidin (Molecular Probes). Live-cell imaging was performed using cells stably expressing Histone-H2B-GFP and Anillin-mCherry constructs under the control of the constitutive act05C promoter. Imaging was performed at room temperature using an Ultraview Vox spinning disc confocal system (PerkinElmer), employing a CSU-X1 scanning unit (Yokogawa) and an Orca-R2 CCD camera (Hamamatsu) fitted to a Leica DMI6000B inverted microscope equipped with a motorized piezo-electric stage (Applied Scientific Instrumentation). Image acquisition was performed using Volocity 6 (Improvision/Perkin Elmer) and a Plan Apo 63X oil immersion objective (NA 1.4) with camera binning set to $2 \times 2$.

\section{Live imaging of human cells.}

HEK293T cells (kind gift from the Marsh lab, UCL) were plated on glass-bottomed dishes (MatTek), HeLa cells stably expressing LifeAct-GFP/Histone-H2B-mRFP (as previously published ${ }^{4,17}$ ) were plated on chambered coverslips (LabTek; Thermo Fisher Scientific) coated with $10 \mu \mathrm{g} / \mathrm{ml}$ fibronectin (Sigma). Before recording of live cells, the medium was changed to phenol-red-free CO2-independent medium L-15 (Leibovitz). For Fig.1c, Fig.3c-d, Extended Data Fig.4a-b and Extended Data Fig.6, frames were acquired at $37{ }^{\circ} \mathrm{C}$ using an UltraView Vox (Perkin Elmer) spinning disc confocal microscope with a $60 \mathrm{X}$ oil objective (NA 1.4) and images acquired using a Hamamatsu C9100-13 EMCCD camera and Volocity software (Perkin Elmer). Images in Fig. 1d and Extended Data Fig.2b were acquired at $37^{\circ} \mathrm{C}$ using a Zeiss Axio Observer Z1 microscope controlled by Volocity 6.3 software (Perkin Elmer) and equipped with an Orca 03GO1 camera (Hamamatsu) and a 40X oil DICII objective (NA 1.3). For the optogenetic experiments (Fig.4d), HEK293T cells were cotransfected with CIBN-GFP-CAAX, Cry2-mCherry, Cry2-mCherry-Sds22 and Sds22-GFP (plasmid from J. Swedlow) using FuGENE HD (Promega). $22 \mathrm{~h}$ after transfection cells were treated with $50 \mathrm{ng} / \mathrm{ml}$ nocodazole for $8 \mathrm{~h}$ and imaged after nocodazole wash-out using a Leica TCS SP5 confocal microscope and 60X oil objective (NA 1.4). Ten consecutive pulses (800ms each) of $488 \mathrm{~nm}$ light at $15 \%$ of 
laser output were used to induce interaction between CIBN and CRY2. 561nm laser was used to image Cry2-mCherry or Cry2-mCherry-Sds22.

\section{Immunofluorescence microscopy in Drosophila.}

Nota from pupae 14-16 h APF were dissected in PBS at room temperature. Tissues were promptly fixed in $10 \%$ trichloroacetic acid or $4 \%$ formaldehyde for $20 \mathrm{~min}$ at room temperature, before being permeabilised in PBS containing 0.1\% triton X-100. Subsequently, nota were incubated in a blocking solution composed of $5 \%$ bovine serum albumin (BSA) and 3\% fetal bovine serum (FBS) (in PBS). Once immunostained and mounted, tissues were imaged using a Leica TCS SP5 confocal microscope and 60X oil objective (NA 1.4).

\section{Immunofluorescence microscopy in human cells.}

For the data shown in Extended Data fig.3f hTERT RPE-1 cells (used previously ${ }^{4}$ ) were arrested in prometaphase by using the Eg5 kinesin inhibitor STLC $(5 \mu \mathrm{M}$, Sigma) for $8 \mathrm{~h}$. Cells were treated with a low doze of nocodazole (20 nM, Sigma) for $3 \mathrm{~h}$ to specifically disrupt astral microtubules ${ }^{33}$. To induce monopolar cytokinesis cells were treated with CDK1 inhibitor RO3306 (10 $\mu \mathrm{M}$, Sigma) for $10 \mathrm{~min}$ and then fixed for $15 \mathrm{~min}$ on ice in $10 \%$ trichloroacetic acid before being processed for immunofluorescence microscopy as described ${ }^{34}$. Images in Extended Data Fig.3f were acquired on a Leica SPE confocal microscope with a 63X lens (NA 1.3).

\section{Immunoprecipitation assays.}

For the data shown in Extended Data Fig.7g dsRNAs targeting LacZ and Sds22 were generated using T7-tagged oligos and a T7 dsRNA kit (Ambion) as previously described $^{35}$. S2 cells were transfected with dsRNA following transfection with PP187B-GFP on the next day. $72 \mathrm{~h}$ after dsRNA transfection cells were lysed in buffer containing $50 \mathrm{mM}$ Hepes $\mathrm{pH} 7.4,150 \mathrm{mM} \mathrm{NaCl}, 0.5 \%$ triton $\mathrm{X}-100$ and a protease inhibitor cocktail (Roche). PP1-87B-GFP was collected with GFP-Trap-A (Chromotek). For the substrate, S2 cells were transfected with FLAG-Moesin. $48 \mathrm{~h}$ after transfection cells were treated with $50 \mathrm{nM}$ Calyculin A for $25 \mathrm{~min}$ and lysed in buffer containing $10 \mathrm{mM}$ Tris $\mathrm{HCl} \mathrm{pH} 7.5,150 \mathrm{mM} \mathrm{NaCl}, 1 \%$ triton $\mathrm{X}-100,0.1 \%$ SDS, 1\% Sodium Deoxycholate, protease inhibitor cocktail (Roche), phosphatase inhibitor cocktails 2 and 3 (Sigma). FLAG-Moesin was collected with Anti-FLAG 
M2 beads (Sigma) and eluted with buffer containing $50 \mathrm{mM}$ Tris $\mathrm{HCl} \mathrm{pH}$ 7.5, $30 \mathrm{mM}$ $\mathrm{NaCl}$ and $150 \mathrm{ng} / \mu \mathrm{L} 3 \times \mathrm{xLAG}$ peptide. Eluted FLAG-Moesin was added to the beads bound to PP1-87B-GFP, incubated at $30{ }^{\circ} \mathrm{C}$ for indicated time and analysed by immunoblotting.

\section{Antibodies and dyes.}

The following antibodies and dyes were used for immunofluorescence microscopy: Rabbit anti-p-ERM (Cell Signaling, 3141S, 1/100 in fly tissues, 1/200 in human cells), Rabbit anti-pMRLC2 (S19) (Cell Signaling, 3671S, 1/100), Mouse anti- $\alpha$ Tubulin (Abcam, DM1 A, 1/100 in fly tissues, 1/200 in human cells), Guinea pig antiCentrosomin (1/1000, a gift from F. Pichaud), DAPI (Molecular Probes, $1 \mu \mathrm{g} / \mathrm{ml}$ ), Phalloidin-TRITC (Sigma-Aldrich). Secondary antibodies from Molecular Probes were conjugated with Alexa488, 546 and 647. The following antibodies were used for western blotting: Rabbit anti-p-ERM (Cell Signaling, 3141S, 1/100), Rabbit antiMoesin (1/25000, a gift from D. Kiehart), Mouse anti-GFP (Roche, No. 11814460001, 1/500), Rabbit anti-Sds22 (Santa Cruz, E-20, 1/100), Mouse anti- $\gamma$ Adaptin (BD Transduction Laboratories, No. 610386, 1/1000) and Mouse antiRacGAP1 (Abnova, M01, 1/50000).

\section{Quantification of protein levels at the cortex.}

FIJI software (http://fiji.sc/Fiji) was used to quantify the actin levels at the cell pole during anaphase (as seen in Fig.1f, Fig.2e and Extended Data Fig.1d). First, a kymograph of the radial cross-section was assembled for each cell (as seen in Fig.2a, b, yellow box denotes the cross-section). Then, the mean actin intensity was systematically measured over time by placing a 9-pixel square region onto the polar cortex at every time-point along the kymographic profile. Values were then normalized against the actin levels in metaphase. To generate the bar graphs of the actin levels, the values obtained for each cell represent the average of 5 values measured during a $50 \mathrm{sec}$ period either before or after the onset of cell elongation.

To quantify the ratios poles/equator (or proximal/distal) of protein levels seen in Fig.1h, Extended Data Fig.1e and Extended Data Fig.3h-i, protein levels were measured by placing a small rectangle onto the polar or equatorial cortex of anaphase cells and retrieving the mean gray value (using FIJI). In Fig.3d, Extended Data 
Fig.2c-d and Extended Data Fig.4a, levels of cortical protein (F-actin or myosin-II) were normalized against cytoplasmic signal. To quantify the levels of F-actin seen in Extended Data Fig.4b and Extended Data Fig.6d, the levels of cortical protein under the chromatin mass were normalized against the levels of cortical signal of the whole cell area (bottom cross-section).

\section{Assembly of plots - actin levels/time and distance DNA-to-cortex/time.}

FIJI software (http://fiji.sc/Fiji) was used to generate kymographic plots of the cortical perimeter over time (as seen in Fig.2g1). A line scan was drawn over the halfperimeter of each Lifeact-GFP-labeled SOP cell during early anaphase ( 9 timepoints, $10 \mathrm{sec}$ resolution). These lines were then straightened and combined in the form of a kymograph (Fig.2g1). These kymographs were saved in a text image format and subsequently binned into 9x9 grids of mean gray values (not shown). These 9x9 grids of actin intensity values were then 'overlaid' to obtain an average plot of actin levels/time for all cells analysed (seen in Fig.2g3). In parallel to this, we measured the DNA-cortex distances d1-9 (as seen in Fig.2f) in each cell during early anaphase, obtaining a 9x9 grid of distance/time as seen in Fig.2g2. Similarly to the actin plots, the distance/time grids of all 10 cells analysed were 'overlaid' to obtain an average plot distance/time (seen in Fig.2g4). Matlab (MathWorks) was used to present the plots aforementioned in the form of color-maps.

\section{Graphs and Statistical Analysis}

Various types of graphs are shown: bar graphs of mean with standard deviation

(Fig.1b, d, f; Fig.2e; Fig.4c; Extended Data Fig.1c-d; Extended Data Fig.2c-d; Extended Data Fig.3c, k; Extended Data Fig.5h, k; Extended Data Fig.7f; Extended Data Fig.9c; Extended Data Fig.10e), scatter plots with median (Fig.3d; Extended Data Fig.1b; Extended Data Fig.3h-i; Extended Data Fig.6c-d) and box-and-whisker plots with 10-90 percentiles (Fig.1h; Extended Data Fig.1e; Extended Data Fig.4a-b; Extended Data Fig.5b, d-e; Extended Data Fig.7c; Extended Data Fig.8c-d). In all cases, Welch's unequal variances $t$-test was used to calculate statistical significance in all analyses. $\mathrm{p}<0.05$ : significant, $\mathrm{p}<0.01$ : very significant, $\mathrm{p}<0.001$ : extremely significant. Linear regression analysis was carried out using Graphpad PRISM. Note, independent data points represent biological replicates: i.e. parallel experiments carried out on genetically identical cells with identical reagents. Figure legends 
indicate when the same primary data (images/movies) were subjected to different types of analysis in parallel. 


\section{Extended Data Figure Legends}

Extended Data Figure 1 - Depletion of RacGAP1 in SOP cells does not affect polar relaxation nor anaphase cell elongation.

a-c, Time-lapse imaging of SOP cells in control and RacGAP1-depleted backgrounds was carried out to analyze the relative timing of polar relaxation and anaphase elongation. We analyzed cells from 5 control animals and 4 RacGAP1 RNAi animals. Representative images are shown in a. White arrowheads indicate actin clearance at the poles. Lifeact-GFP was used to label F-actin. For control (19 cells) and RacGAP1 (16 cells) cells that formed a furrow, the time period between anaphase onset and furrow initiation or furrow completion is plotted in $\mathbf{b}$ (black lines denote the median). Cell length was plotted in c for the control (18 cells), and for RacGAP1 RNAi cells that were delayed in furrow formation ( 15 cells) or that failed to form a furrow (5 cells). d, Relative levels of polar actin were compared across movies of 12 control RNAi cells from 4 animals (same as seen in Fig.2e) and from 12 RacGAP1 RNAi cells from 3 animals. e, Graph shows the ratio of levels of cortical actin at poles versus the equator at mid anaphase for 20 control cells from 5 animals, and for 13 RacGAP1 RNAi cells from 3 animals; Data are shown as mean \pm standard deviation in $\mathrm{c}$ and $\mathrm{d}$, and box-and-whisker plots with $10-90$ percentiles in e. Scale bars $=5 \mu \mathrm{m}$. A two-tailed unpaired t-test was used to calculate statistical significance; $\mathrm{P}>0.05$ was deemed not significant.

Extended Data Figure 2 - Depletion of RacGAP1 impairs Myosin-II equatorial accumulation and furrow ingression, but does not affect actin clearance from the poles at mid-anaphase.

a, Western blot showing RacGAP1 depletion in HeLa cells. b, Representative DIC stills from movies show HeLa cells at indicated times after the onset of anaphase. Images show control cells before and after furrow initiation and RacGAP1-depleted cells at mid-anaphase - since all fail cytokinesis. Arrowheads point to blebbing. c-d, Graphs show levels of cortical myosin-II (c) and cortical actin (d) in the polar and equatorial regions of cells. Levels were measured in HeLa cells expressing Myosin-IIGFP/Utrophin-Ruby treated with Control siRNA (15 cells, 3 experiments) or siRacGAP1 siRNA (oligonucleotide \#4, seen in a) (15 cells, 3 experiments). Data are shown as mean \pm standard deviation. e-f, Representative images and corresponding kymographs taken from 12 time-lapse movies of fly SOP cells fluorescently labeled 
for both myosin-II (Sqh-mCherry) and actin filaments (GMA) undergoing anaphase. Note that the same cells were used for the analysis in ED Figure 9a. Anaphase onset $=0 \mathrm{sec}$. Asterisks mark the chromosomes. Kymographs of anaphase progression of the E-P perimeter section depicted in e. Note that actin and myosin-II show different redistribution patterns at the cortex during anaphase (also, see white arrowheads in e). g, Fly epithelial cell at mid-anaphase immunostained for p-myosin-II, F-actin (phalloidin), Tubulin and DNA, representative of 3 cells. h, Fly epithelial cell at midanaphase immunostained for p-Moesin and DNA, representative of 15 cells; Scale bars in $\mathrm{b}, \mathrm{e}, \mathrm{g}, \mathrm{h}=5 \mu \mathrm{m}$. A two-tailed unpaired t-test was used to calculate statistical significance.

\section{Extended Data Figure 3 - Actin clearance from the poles is independent of centrosomes and astral microtubules.}

a, SOP cell imaged at metaphase/anaphase (left) (representative of 3 imaged precisely in this way), together with kymograph of cross-section (yellow box). Cnn indicates centrosomin. Lifeact-GFP was used to label F-actin. b-c, Fly epithelial cells were fixed and immunostained for centrosomin, Tubulin and DNA. Green arrowheads indicate the presence of centrosomes in control cells. Representative images are shown (b) together with quantification of the relative centrosomin levels at the centrosome (c) for 25 cells from 3 control animals and for 26 cells from $3 \mathrm{Asl}^{\mathrm{mecD}}$ animals. A two-tailed unpaired t-test indicated that there was a significant difference in centrosomal Cnn levels in the two cases. d, Scheme of SOP cells dividing in different orientations. A-P axis $=0^{\circ}$ (left). Rosette plots indicate spindle axis angle measured at the onset of anaphase for 34 control cells from 3 animals and for 23 $\mathrm{Asl}^{\text {mecD }}$ cells from 3 animals. e, Time-lapse stills of SOP cells expressing GMA to label F-actin taken at early and mid-anaphase in control (representative of 12 cells) and $\mathrm{Asl}^{\mathrm{mecD}}$ (representative of 16 cells) mutant backgrounds (as shown in Fig.1e-f), together with plot profiles (right) denoting the relative actin levels across the cell. Asterisks mark the chromosomes. f, Images show representative STLC-treated RPE-1 cells treated with or without nocodazole $20 \mathrm{nM}$ and/or RO3306 (15 cells were analysed for each condition), fixed and stained for pERM proteins, DNA and Tubulin. g, Images in top panel show representative Mad2-depleted S2 cells treated with 25 $\mu \mathrm{M}$ colchicine and stained for F-actin (phalloidin) and Anillin from a population of 
13 cells. Similarly, bottom panel shows images of S2 cells (representative of 13 cells) treated with colchicine and forced to exit mitosis with $20 \mu \mathrm{M}$ RO3306, and stained for F-actin (phalloidin) and p-Moesin (pERM antibody). h-i, Ratio proximal/distal of levels of cortical F-actin (h) and p-Moesin (i) (refers to g). Mean is labeled in red. j, S2 cells expressing either H2B-GFP/Anillin-Cherry or Lifeact-GFP/H2B-Cherry were imaged during mitotic exit. Representative stills and the corresponding kymographs are shown in $\mathrm{j}$ (equivalent to Phenotype $\mathrm{I}$ in $\mathrm{k}$ ). Top panel, $\mathrm{n}=68$ cells, 3 experiments. Bottom panel, $\mathrm{n}=24$ cells, 1 experiment. $\mathbf{k}$, Phenotypic quantification of Anillin-Cherry-expressing S2 cells treated with colchicine and forced to exit mitosis through either Mad2 depletion (as depicted in j, top panel) or through treatment with RO3306. Bar graphs depict mean and standard deviation. Phenotype I: DNA and cortex are polarized. Phenotype II: neither DNA nor cortex is polarized. Phenotype III: DNA is polarized but cortex is not. Mad2 RNAi, $\mathrm{n}=68$ cells, 3 experiments. $\mathrm{RO} 3306, \mathrm{n}=121$ cells, 2 experiments. Scale bars $=5 \mu \mathrm{m}$.

\section{Extended Data Figure 4 - Anaphase DNA-induced clearance of cortical F-actin.}

a-b, Data show representative stills and corresponding quantitative data extracted from movies of 17 STLC-treated HeLa cells (from 3 independent experiments) expressing LifeAct-GFP and H2B-Cherry forced to flatten through Rap1* expression before and after treatment with the CDK inhibitor, RO3306. a, Images show XZ cross-section of a representative flattened HeLa cell, before and after treatment with RO3306. Levels of cortical F-actin above the chromatin were normalised against cytoplasmic fluorescence signal (ratios are shown in green on right). Graph on right shows normalised levels of cortical actin overlying the DNA pre- and post-treatment with RO3306 (at 6 min after drug addition) for all 17 cells. b, XY cross-sections of representative cell shown in a (left). Levels of cortical actin below the chromatin (see dotted region) were normalised against the fluorescence signal in the most basal confocal-section (ratios shown in green on right). Graph on right shows normalised levels of basal cortical actin lying beneath the DNA for all 17 cells. c-d, Scheme and data to test the correlation between cell elongation and anaphase chromosome movements at the anterior pole of fly SOP cells. c, Scheme depicts distances D1, D2 and D3. d, Graph shows D1, D2 and D3 plotted for anterior pole during anaphase for representative SOP cell A (shown in Fig. 2a-b, 1 of 12 analysed). e-i, Experiments to test how cortical actin is cleared from the anterior and posterior cortex of $10 \mathrm{SOP}$ 
cells during chromosome segregation. e, Scheme of cortical regions c1-9 (as seen in Fig.2f). f-g, Stills of the posterior and anterior poles of representative SOP cell imaged in early anaphase. Arrowheads point to poor and strong actin clearance, respectively. h-i, Average plot of cortical actin measured over time for the posterior pole and anterior pole (same as seen in Fig. $2 \mathrm{~g} 3$ ). The F-actin threshold level was set to 3.0 to allow a better comparison between anterior and posterior poles. These data show that clearance of actin on the anterior pole occurs prior to posterior pole in SOP cells. Scale bars in $\mathbf{f}$ and $\mathbf{g}=5 \mu \mathrm{m}$. Box-and-whisker plots show median together with 10-90 percentiles. A two-tailed unpaired t-test was used to calculate statistical significance.

\section{Extended Data Figure 5 - Depletion of PP1-87B or Sds22 impairs cell elongation in SOP cells.}

a-e, The correlation between cell elongation and the approach of chromatin to the cortex was analysed in control (12 cells, 4 animals), PP1-87B (16 cells, 4 animals) and Sds22 (10 cells, 3 animals) RNAi cells. a, Plot of the distance DNA-to-cortex during anaphase in 3 representative SOP cells in control, PP1-87B RNAi and Sds22 RNAi backgrounds. Anterior pole depicted. $\mathrm{mD}$, mean distance during anaphase. $\mathbf{b}$, Boxplot of mean distance DNA-to-cortex in mid-anaphase. c, Graphs show distance from cell center-pole plotted before and after elongation onset in representative cells for each of the 3 conditions (in black), together with a fitted linear regression (in red). d-e, Boxplot to show the slopes of linear regression analysis (as in c) before the elongation onset and after the elongation onset for control, PP1-87B RNAi and Sds22 RNAi backgrounds. f-h, Pre-furrow anaphase elongation for control (22 cells from 5 animals), PP1-87B (21 cells from 4 animals) and Sds22 (14 cells from 3 animals) RNAi SOP cells expressing Lifeact-GFP. f, Representative images of cells. g, Outlines of the boundary of cells shown in $f$ at different times following the onset of anaphase. h, Graph shows pre-furrow anaphase cell elongation for cells in each background. These data show that upon PP1-87B or Sds22 depletion, SOP cells display faulty anaphase elongation. i-k, Analysis shows anaphase elongation in control (12 cells from 3 animals) and $\mathrm{Asl}^{\mathrm{mecD}}$ mutant (16 cells from 3 animals) cells. i, Images show F-actin in representative SOP cells expressing GMA in control and $\mathrm{Asl}^{\text {mecD }}$ mutant backgrounds. j, Outlines of boundary at different times following anaphase onset for representative cells shown in i. $\mathbf{k}$, Plot of cell elongation in the 
backgrounds seen in $\mathrm{d}$. These data show that anaphase cell elongation is not perturbed in the absence of centrosomes or astral microtubules. $n$, number of cells. Control, 3 animals. Asl ${ }^{\mathrm{mecD}}, 3$ animals. Scale bars $=5 \mu \mathrm{m}$. Box-and-whisker plots show median and 10-90 percentiles. Bar charts show mean and standard deviation. Significance was assessed using a two-tailed unpaired t-test. $\mathrm{p}>0.05$ was deemed not significant (n.s.).

\section{Extended Data Figure 6 - Depletion of Sds22 in human cells leads to impaired} clearance of cortical actin.

a, Western blot showing depletion of Sds22 in HeLa cells through RNA interference. b-c, Control (representative of 18 cells) and Sds22 RNAi (representative of 11 cells) STLC-treated HeLa cells expressing Lifeact-GFP and H2B-mCherry before and after RO3306 treatment (which forces cells to exit mitosis), together with a box and whisker plot (median and 10 and $90^{\text {th }}$ percentile) quantifying of the minimal DNA-tocortex distance after treatment with RO3306 in each case. d, Box and whisker plot (median and 10 and $90^{\text {th }}$ percentile) showing cortical F-actin clearance below the DNA (as seen in EDfig.4b); siRNA oligonucleotide \#5 (seen in a) was used in experiments shown in b-d and Fig.3c-d. Scale bar in $b=5 \mu \mathrm{m}$. Significance was assessed using a two-tailed unpaired t-test.

\section{Extended Data Figure 7 - Moesin is a target of PP1-87B/Sds22 and controls cortical relaxation at anaphase.}

a-c, The effect of constitutively active Moesin on anaphase polar relaxation. a, An SOP cell ( 1 of 13 cells) expressing constitutively active Moesin (MoesinT559D) imaged in metaphase and anaphase (top) and kymograph of cross-section over time (yellow box). b, Plot of the distance DNA-to-cortex over time for representative Moesin-GFP cell and a MoesinT559D-GFP-expressing cell (Cell Y in a). mD, mean distance during anaphase. Only anterior pole indicated. c, Box and whisker plot (median and 10/90 percentiles) of $\mathrm{mD}$ in Moesin-GFP and MoesinT559D-GFPexpressing cells. This shows that the DNA comes into close apposition to the cortex in cells expressing constitutively active Moesin as the result of a failure to trigger efficient polar relaxation, as it does in cells depleted for PP1-87B or Sds22. d-f, The effect of TD-Moesin expression on pre-furrow elongation in the same experiment as a-c. d, Images show representative SOP cells expressing GFP-Moesin or GFPPMoesinTD transgenes at metaphase and anaphase (out of 13 cells in each case). e, 
Outlines of the boundary of cells shown in $\mathrm{d}$ at different times during anaphase. $f$, Plot of cell elongation in these two backgrounds showing mean and standard deviation. As observed in PP1-87B or Sds22-depleted cells, MoesinT559D-GFP expressing cells show aberrant cell elongation at anaphase. g, Immunoprecipitation assays showing Moesin dephosphorylation by PP1-87B/Sds22. CalA (calyculin A) is an inhibitor of PP1 activity (left panel). Upon addition of CalA, PP1-87B activity is suppressed, leading to higher levels of phosphorylated Moesin than in the absence of compound (see p-FLAG-Moesin immunoblotting). PP1-87B acts with Sds22 to dephosphorylate active Moesin (see right panel in $\mathbf{g}$ ). Upon Sds22 depletion, PP1-87B is less efficient in inactivating Moesin. Red arrows indicate PP1-87B-GFP band. Results in $g$ were replicated 3 times. h, Scheme of PP1/Sds22-dependent inactivation of Moesin. Scale bar in a and $d=5 \mu \mathrm{m}$. Significance was assessed using a two-tailed unpaired t-test.

Extended Data Figure 8 - Depletion of PP1-87B and Sd22, or expression of MoesinT559D-GFP all lead to severe shape defects in telophase cells.

a-d, Data show the impact of silencing PP1-87B RNAi or Sds22 and of overexpressing TD-Moesin on telophase cell shape. a, Stills show representative telophase cells in control (1 from 32), PP1-87B RNAi (1 from 31) and Sds22 RNAi (1 from 27) backgrounds. Circularity of cells, C, is indicated. F-actin is labeled by Lifeact-GFP. b, Images show representative stills of telophase Moesin-GFP (1 from 19) or MoesinT559D-GFP (1 from 15) cells. Circularity of cells, C, is indicated. PIIa and PIIb are the cells that result from an asymmetric SOP division (in a and b). c, Boxplot of circularity of nascent cells at telophase in control, PP1-87B RNAi and Sds22 RNAi tissues. d, Boxplot of circularity of nascent cells at telophase in Moesin-GFP and MoesinT559D-GFP expressing tissues. Scale bar $=5 \mu \mathrm{m}$. Box and whisker plots show median and 10/90 percentiles. Significance was assessed using a two-tailed unpaired t-test.

\section{Extended Data Figure 9 - Polarization of cortical Myosin-II in anaphase does not depend on PP1 phosphatase.}

a-d, Data show the impact of PP1-87B silencing (16 cells from 4 animals) on Myosin repolarization during anaphase onset relative to a control (12 cells from 4 animals). ab, Stills of representative control (a) and PP1-87B-depleted (b) SOP cells in anaphase 
labeled for myosin-II (Sqh-mCherry) and F-actin (GMA) (top), together with the corresponding kymographs showing the E-P perimeter section during anaphase progression. c, Schematic and graph show length of actin and myosin-II domains along the E-P perimeter in control and PP1-87B RNAi SOP cells. Mean and standard deviation are shown. Significance was assessed using a two-tailed unpaired t-test. $\mathrm{p}>0.05$ was deemed not significant (n.s.). Scale bar $=5 \mu \mathrm{m}$. n, number of cells. These data show that PP1-87B, while controlling the polarisation of cortical actin in anaphase, it does not affect the timely accumulation of myosin-II at the equator.

\section{Extended Data Figure 10 - Local accumulation of Sds22 triggers polar blebbing} in anaphase.

a, Confocal cross-sections of a representative anaphase epithelial cell showing colocalisation of Sds22 and the kinetochore protein $\operatorname{Spc} 25$ ( 1 of 10 cells). Insets of regions pointed by arrowheads. b-e, Data assess the impact of KNL1 silencing on Sds22-GFP localization and polar relaxation. b-c, Representative epithelial cell expressing Sds22-GFP imaged during anaphase, together with corresponding kymograph of anaphase progression. Black arrowheads point to polar blebbing. Inverted lookup table in b-c. Darker tone indicates stronger GFP signal. Scale bar in $\mathbf{a}-\mathbf{b}=5 \mu \mathrm{m}$. d, Line scans across kinetochore regions denoted by the green arrowheads in representative images shown in Fig.4a. e, Levels of Sds22-GFP at kinetochores in control (17 cells from 4 animals) and KNL1 RNAi cells ( 9 cells from 3 animals) normalised against cytoplasmic GFP signal. Graphs show mean and standard deviation. Significance was assessed using a two-tailed unpaired t-test. f, Graphic representation of the blue light-induced cryptochrome-based protein-protein interaction system underlying the data shown in Fig.4d. This scheme shows how CRY2-tagged Sds22 subunit interacts with membrane-tethered CIBN upon blue light irradiation, promoting fast translocation of the phosphatase to the plasma membrane and inactivation of cortical Moesin and, consequently, abrogation of F-actin linkage to the membrane.

Supplementary Video 1 - Live imaging of an SOP cell labeled for F-actin (gray) and DNA (red) from anaphase onset (0 sec) until mid-anaphase. Polar relaxation occurs when the DNA masses come into close apposition with the cortex. Scale bar $=5 \mu \mathrm{m}$. (This video refers to Fig.2a-b) 
Supplementary Video 2 - Live imaging of a PP1-87B-depleted SOP cell labeled for F-actin (gray) and DNA (red) from anaphase onset $(0 \mathrm{sec})$ until mid-anaphase. Scale bar $=5 \mu \mathrm{m}$. (This video refers to Fig.3a-b)

Supplementary Video 3 - Live imaging of an Sds22-depleted SOP cell labeled for Factin (gray) and DNA (red) from anaphase onset (0 sec) until mid-anaphase. Scale bar $=5 \mu \mathrm{m}$. (This video refers to Fig.3a-b)

Supplementary Video 4 - Live imaging of an SOP cell labeled for F-actin (green) and DNA (red) from anaphase onset $(0 \mathrm{sec})$ until late telophase. Scale bar $=5 \mu \mathrm{m}$. (This video refers to Extended Data Fig.8a)

Supplementary Video 5 - Live imaging of a PP1-87B-depleted SOP cell labeled for F-actin (green) and DNA (red) from anaphase onset (0 sec) until late telophase. Scale bar $=5 \mu \mathrm{m}$. (This video refers to Extended Data Fig.8a)

Supplementary Video 6 - Live imaging of an Sds22-depleted SOP cell labeled for Factin (green) and DNA (red) from anaphase onset $(0 \mathrm{sec})$ until late telophase. Scale bar $=5 \mu \mathrm{m}$. (This video refers to Extended Data Fig.8a)

Supplementary Video 7 - Live imaging of an SOP cell expressing MoesinT559DGFP (green) from anaphase onset $(0 \mathrm{sec})$ until late telophase. Scale bar $=5 \mu \mathrm{m}$. (This video refers to Extended Data Fig.8b). 


\section{Supplementary References}

28 Edwards, K. A., Demsky, M., Montague, R. A., Weymouth, N. \& Kiehart, D. P. GFP-moesin illuminates actin cytoskeleton dynamics in living tissue and demonstrates cell shape changes during morphogenesis in Drosophila. Dev Biol 191, 103-117, doi:S0012-1606(97)98707-8 [pii]

10.1006/dbio.1997.8707 (1997).

29 Mummery-Widmer, J. L. et al. Genome-wide analysis of Notch signalling in Drosophila by transgenic RNAi. Nature 458, 987-992, doi:nature07936 [pii]

10.1038/nature07936 (2009).

30 Matsumoto, K., Toh-e, A. \& Oshima, Y. Genetic control of galactokinase synthesis in Saccharomyces cerevisiae: evidence for constitutive expression of the positive regulatory gene gal4. J Bacteriol 134, 446-457 (1978).

31 Jeong, J. Y. et al. One-step sequence- and ligation-independent cloning as a rapid and versatile cloning method for functional genomics studies. Appl Environ Microbiol 78, 5440-5443, doi:AEM.00844-12 [pii]

10.1128/AEM.00844-12 (2012).

32 Hickson, G. R. \& O'Farrell, P. H. Rho-dependent control of anillin behavior during cytokinesis. J Cell Biol 180, 285-294, doi:jcb.200709005 [pii]

10.1083/jcb.200709005 (2008).

33 Thery, M. et al. The extracellular matrix guides the orientation of the cell division axis. Nat Cell Biol 7, 947-953, doi:ncb1307 [pii]

10.1038/ncb1307 (2005).

34 Lenart, P. et al. The small-molecule inhibitor BI 2536 reveals novel insights into mitotic roles of polo-like kinase 1. Curr Biol 17, 304-315, doi:S09609822(07)00831-7 [pii]

10.1016/j.cub.2006.12.046(2007).

35 Liu, T., Sims, D. \& Baum, B. Parallel RNAi screens across different cell lines identify generic and cell type-specific regulators of actin organization and cell morphology. Genome Biol 10, R26, doi:gb-2009-10-3-r26 [pii]

10.1186/gb-2009-10-3-r26 (2009). 

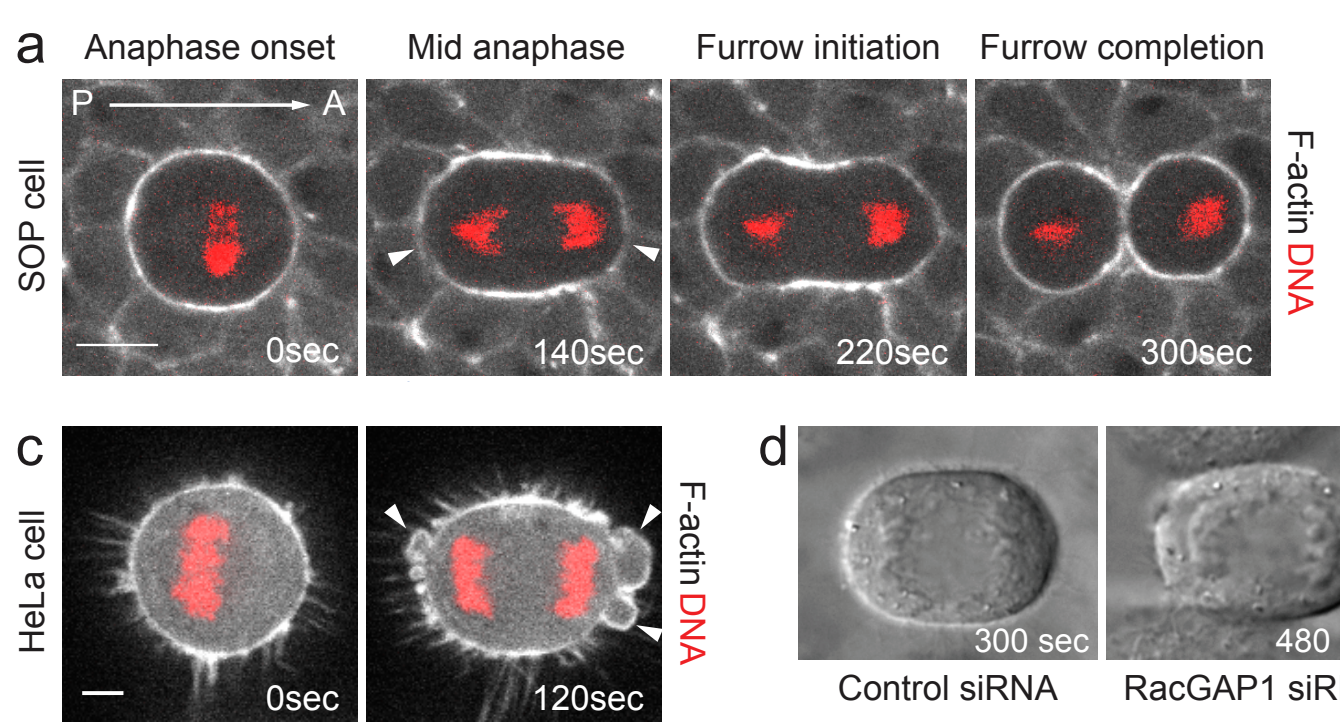

Control siRNA
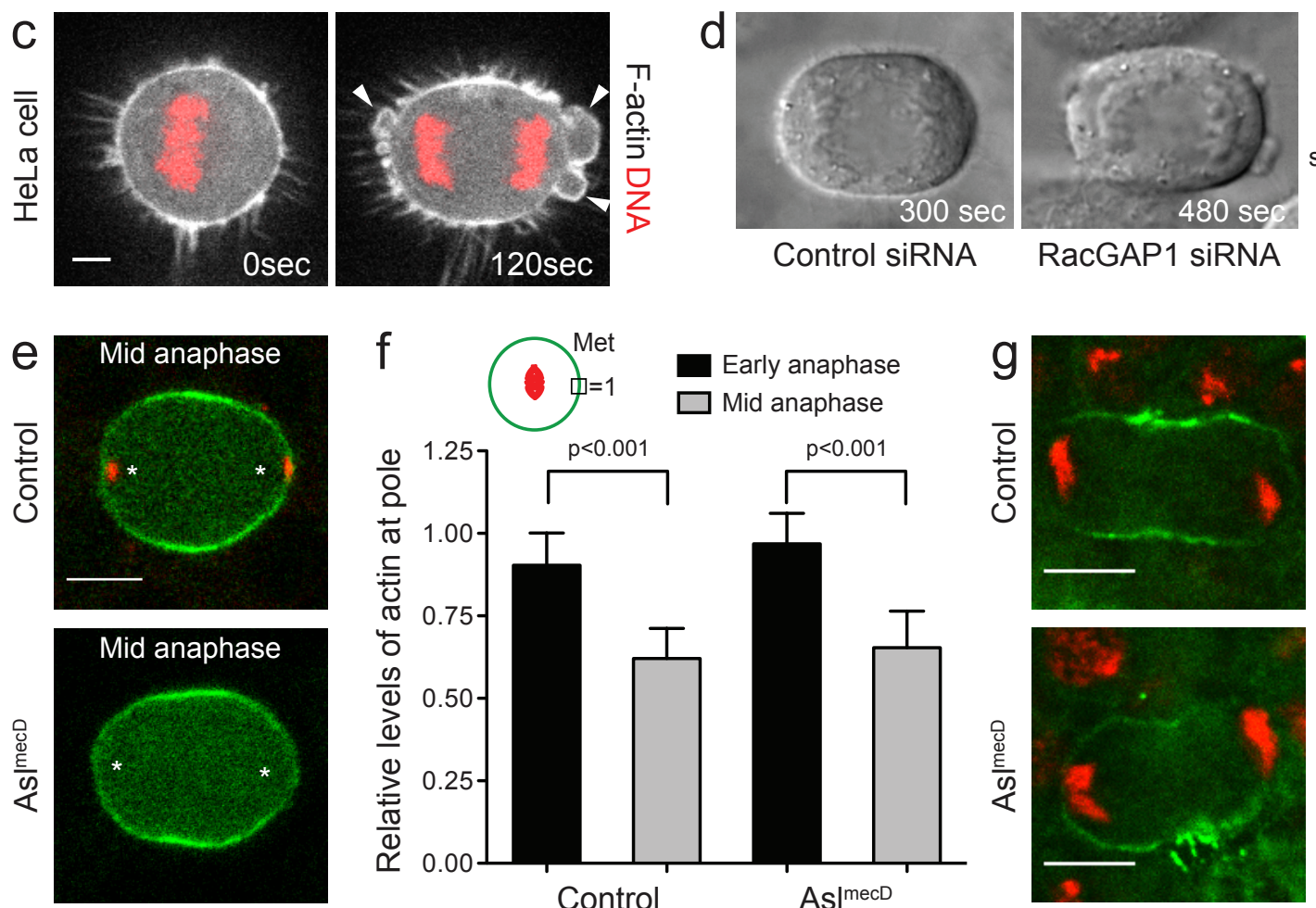

F-actin Centrosomin
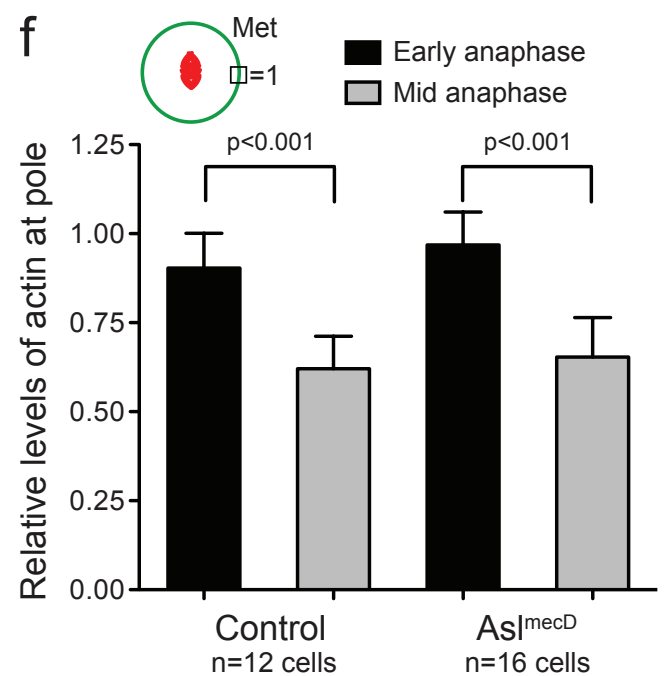

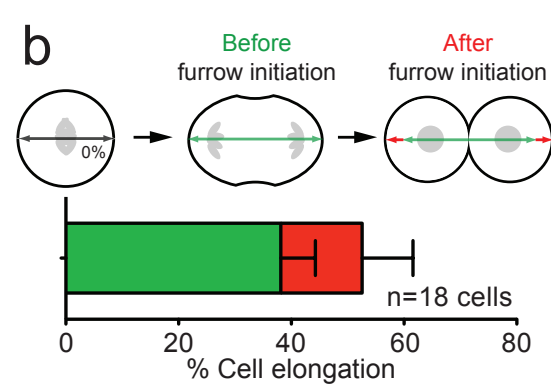

Metaphase $\square$ Anaphase
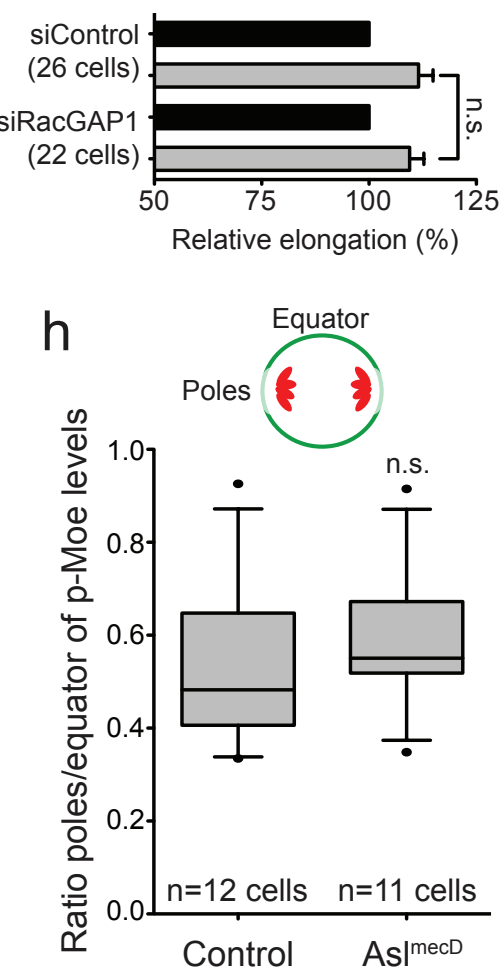


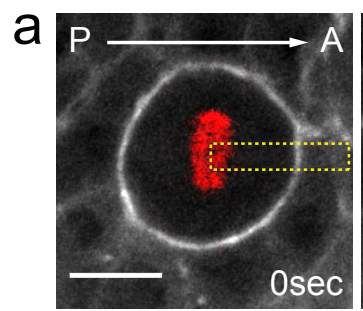

F-actin DNA

b Time after anaphase onset (sec)

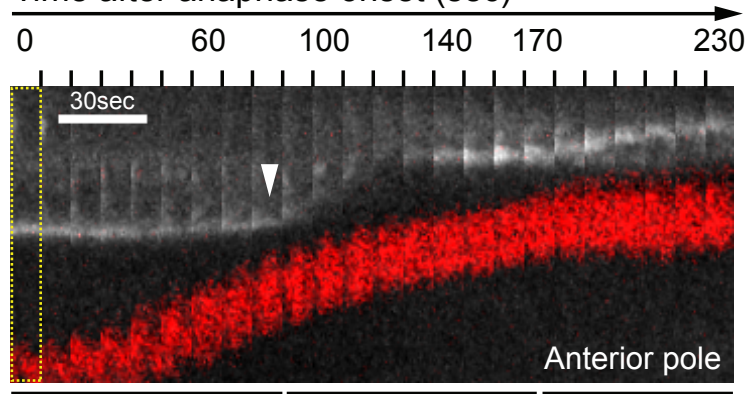

Early ana
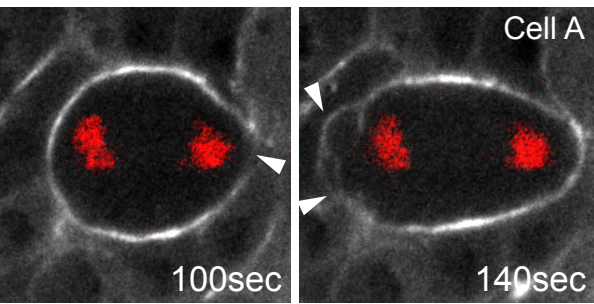

$149 \sec$
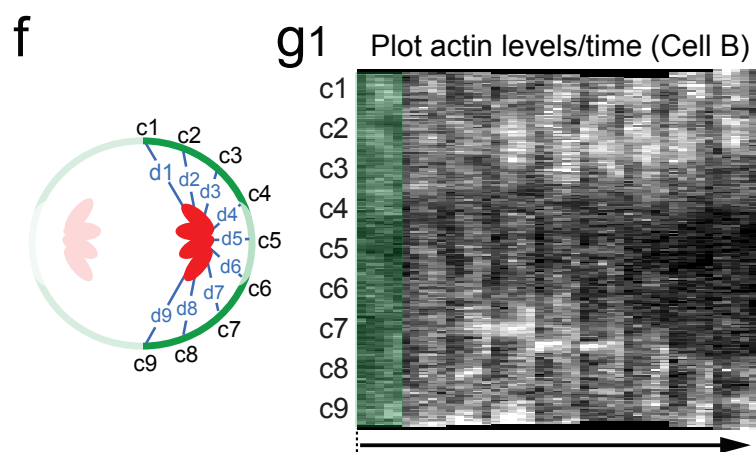

Time to pole expansion

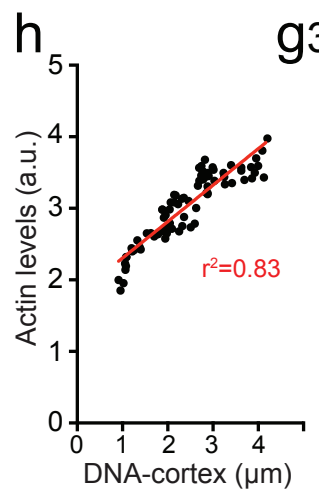

g3

3 Average plot actin levels/time

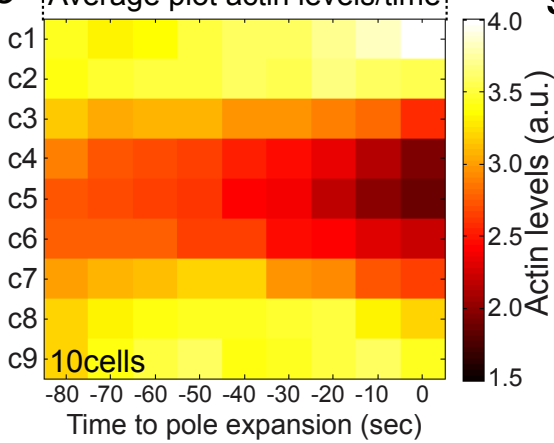

C

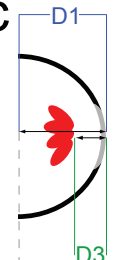

D3

Cell center
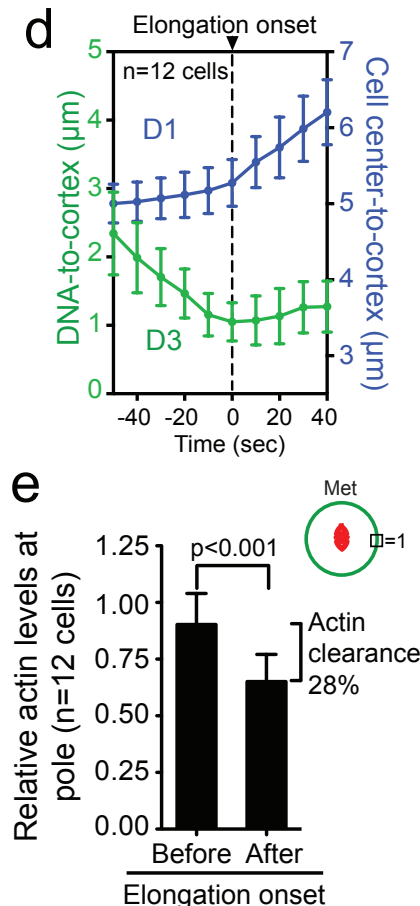

g2 Plot distance/time (Cell B)

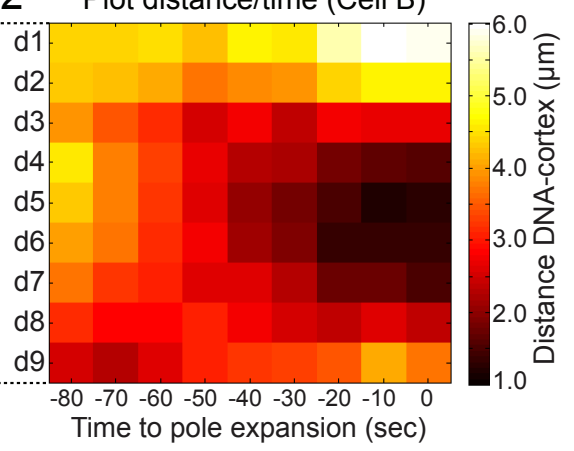

g4 Average plot distance/time

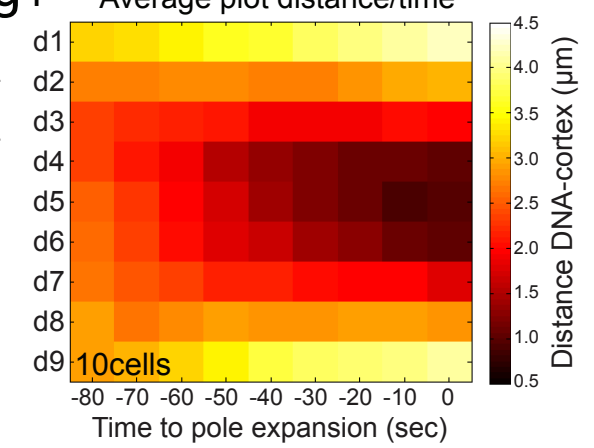



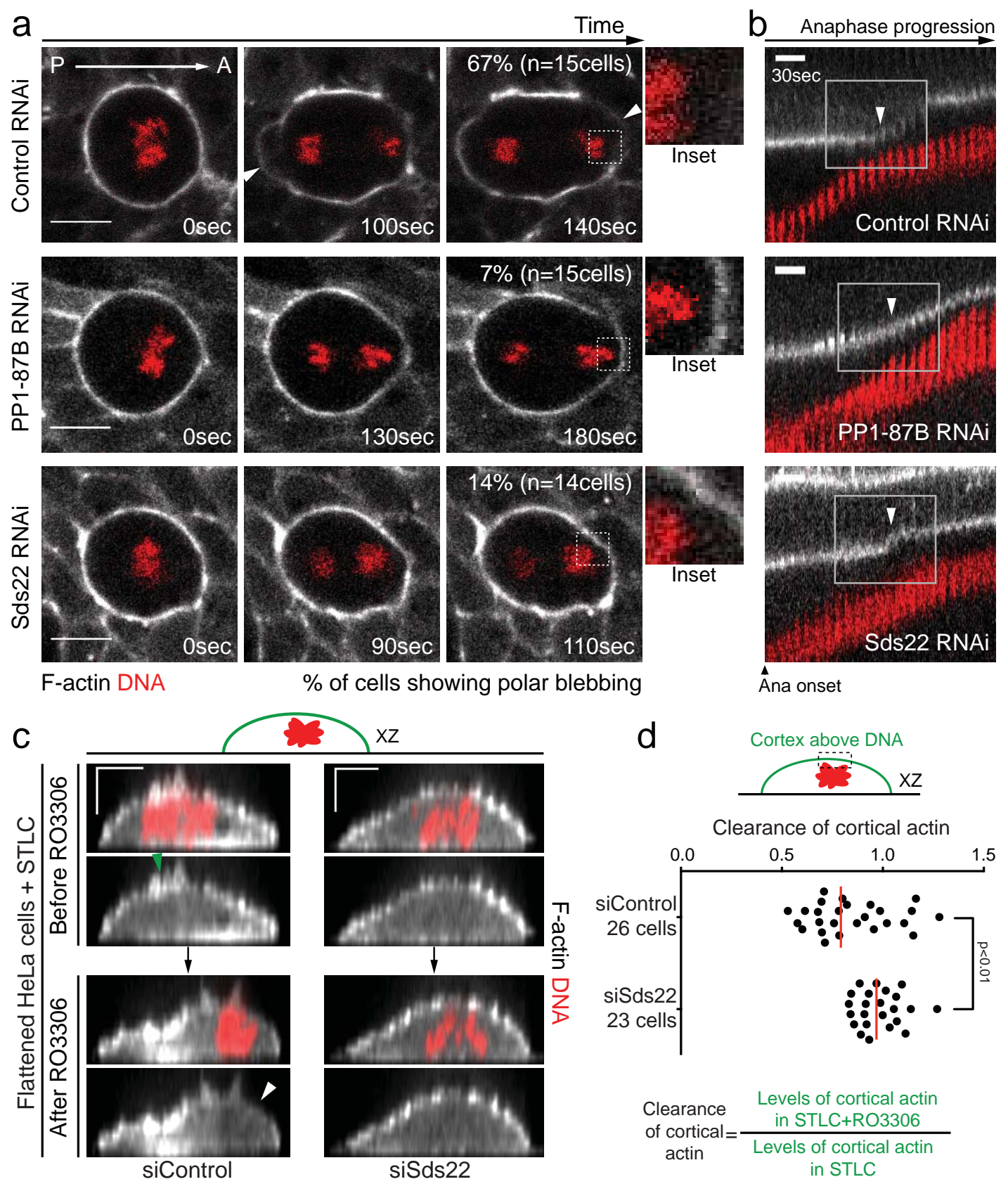

Clearance of cortical actin

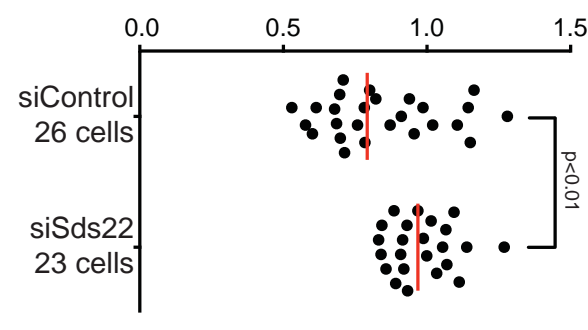

$\begin{aligned} & \text { Clearance } \\ & \text { of cortical } \\ & \text { actin }\end{aligned}=\frac{\begin{array}{c}\text { Levels of cortical actin } \\ \text { in STLC+RO3306 }\end{array}}{\begin{array}{c}\text { Levels of cortical actin } \\ \text { in STLC }\end{array}}$ 

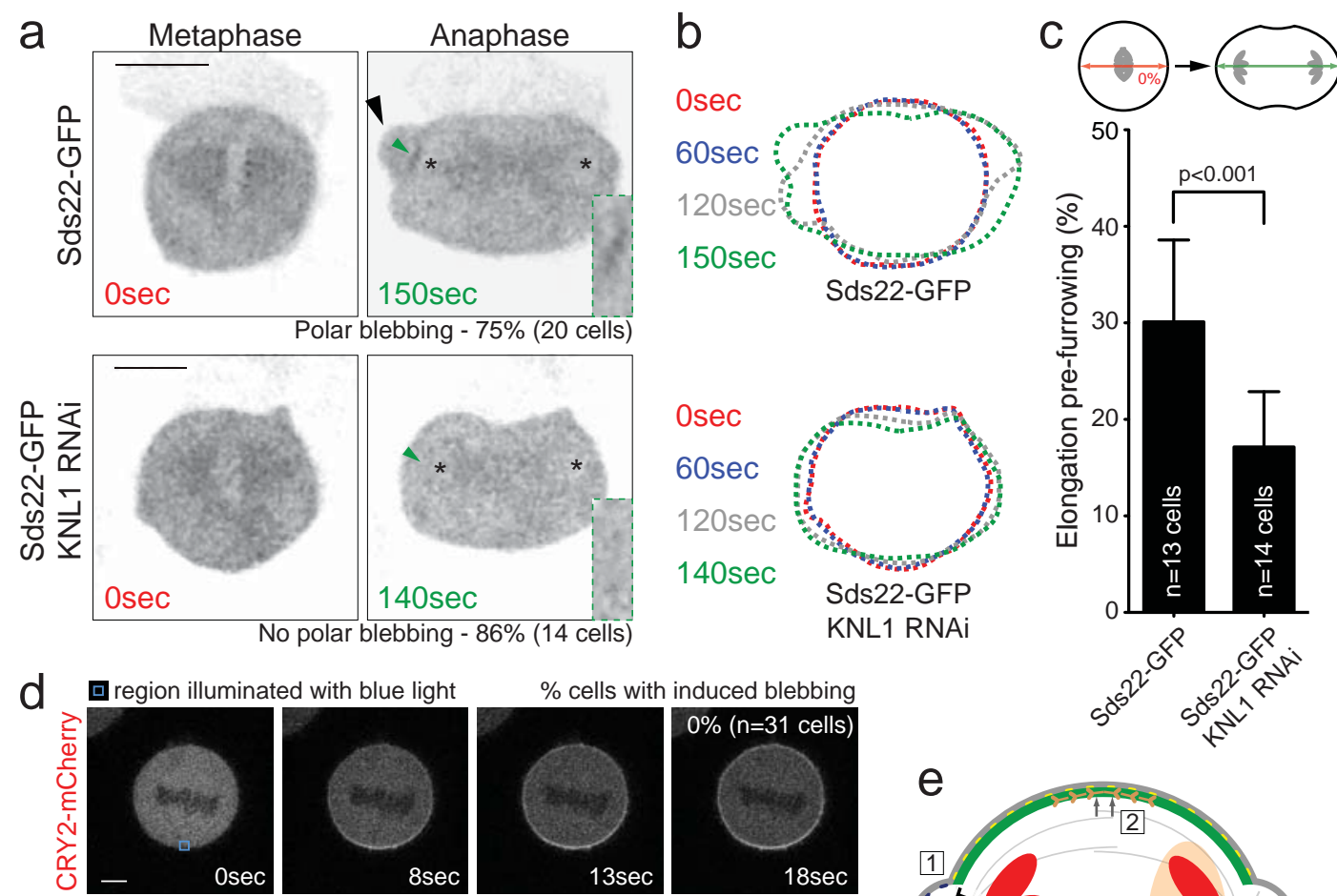

$\%$ cells with induced blebbing
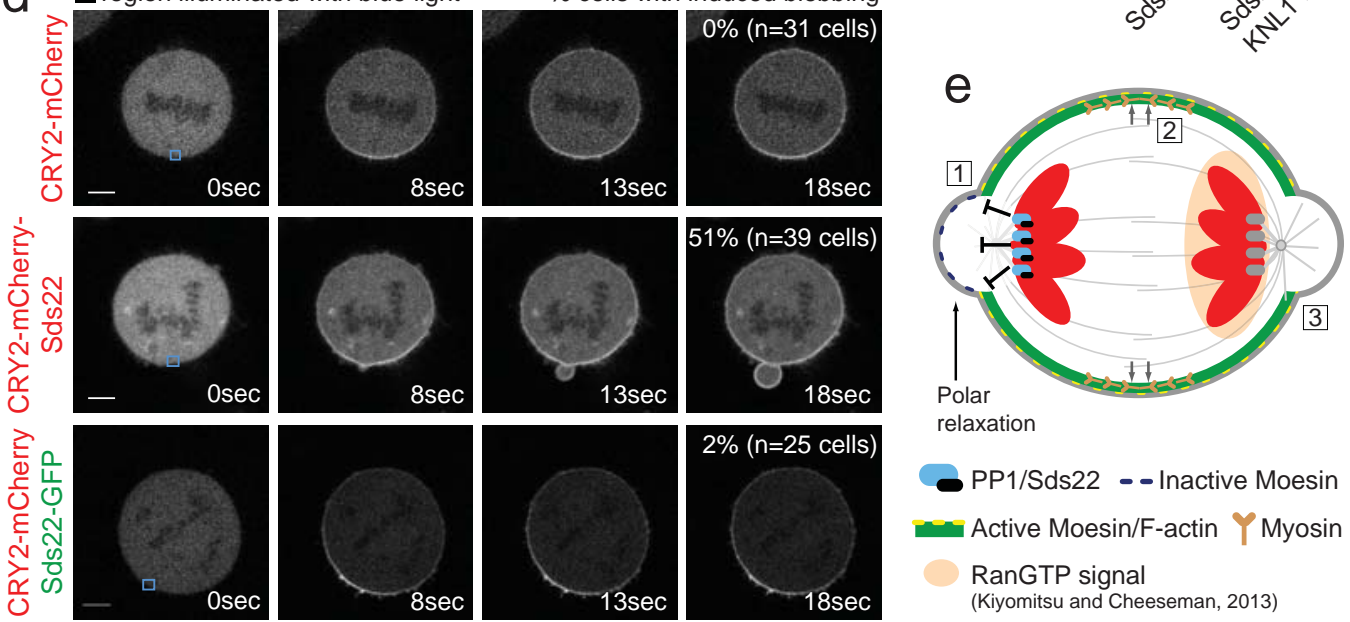

$2 \%(n=25$ cells $)$

PP1/Sds22 _ - Inactive Moesin

Active Moesin/F-actin Y Myosin II

RanGTP signal

(Kiyomitsu and Cheeseman, 2013) 